\title{
JD22
}

\section{Astronomy from the Moon}

\author{
Chairperson and Editor: Y. Terzian
}




\title{
THE ROLE OF LUNAR ASTRONOMY IN THE EXPLORATION AND DEVELOP- MENT OF THE MOON
}

\author{
W. I. MCLAUGHLIN \\ Jet Propulsion Laboratory \\ 4800 Oak Grove Drive, Pasadena, California 91109-8099, USA
}

\begin{abstract}
Lunar astronomical facilities can be robotically emplaced or form an adjunct to a human base. Connected facilities are a means to increase science-per-dollar. Near-Earth and Centaur asteroids may also serve as venues for astronomy.
\end{abstract}

\section{Introduction}

The idea of using the Moon as a location for astronomical facilities is of long standing. Robert S. Richardson, a former Mount Wilson astronomer, wrote a piece, "Astronomical Observations from the Moon", in 1947 [1] wherein he demonstrated a sound grasp of the advantages to be gained from a lunar site, but some of his concerns show how far the relevant technology has advanced in 50 years. "A pendulum clock which keeps accurate time upon the earth would lose at an alarming rate upon the moon, since the acceleration of gravity there is one-sixth that upon the earth." The objective of this paper is to asses the potential of the Moon as a venue for conducting investigations in astronomy. The assesment falls naturally into two cases: 1) where the emplacement of a facility is done solely through robotic means, and 2) where the agency of human effort is employed on the Moon. Considerable thought has been given, particulary in the last 15 years, to lunar astronomy. A view of this contained in four symposia (see [2] through [5]), and ESA [6] and NASA [7] reports. A note is appended with respect to extending the concepts to asteroids.

\section{Robotic Emplacements}

A key criterion is whether a mission is competetive with free-flyer counterparts. To date, the leading lunar candidate may be a low-frequency $(<20 \mathrm{MHz})$ radio array. Lunar far-side, a quiet zone, is to be preferred, but a near-side location is also of value for emplacement of this set of dipole antennas [7]. One-meter telescopes in the ultraviolet or infrared or an optical interferometric array may not be quite competetive with free-flyers [8] but might be when part of their cost is allocated to site selection and technology validation for a subsequent lunar scientific base. The cost of robotic lunar emplacements could be decreased by partial inheritance of physical assets from one mission to the next; this strategy would increase the science-per-dollar. The dependence is that of one investigationn upon either temporal predecessors or contemporary emplacements [9].

Two issues must be addressed: 1) Can schemes for inheritance be formulated? and 2) Can engineering systems survive long on the Moon, particulary with a diurnal thermal cycle of about $300 \mathrm{~K}$ at the equator? (Polar regions have a smaller cycle, $20 \mathrm{~K}$ or less). There are two parts to issue 1: designs for reusability (the "inheritance") and the economics of resuability. The first part is basically a question of connectivity and will only be adressed by listing some functional examples: instrument change-out or augmentation; inherited rover services; passive thermal control (shade!); inherited data systems. See Fig.1 for an idealized sketch of the economic concepts. 


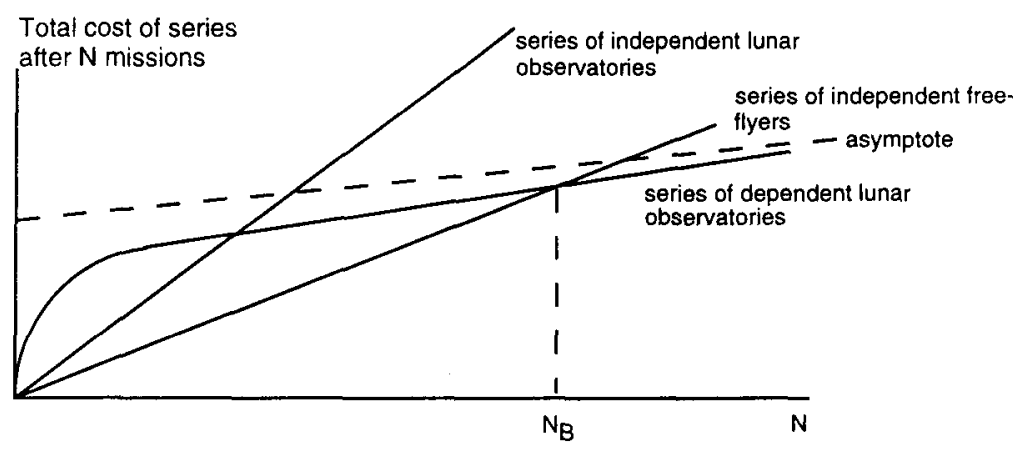

Figure 1. The point $\mathrm{N}_{\mathrm{B}}$ is the "break-even" number. Adaptations for connectivity and survivability account for the initial higher cost of the dependent series. It is possible that $5 \leq \mathrm{N}_{\mathrm{B}} \leq 20$.

\section{Human-Assisted Emplacements}

If a human base is established on the Moon, the selection of astronomical investigations depends upon scientific priorities, budgets, and the support available from the lunar base. In the absence of details, one can only speculate. Assume that the gravity-well and thermal-cycling problems have been ameliorated due to the existence of a transportation system and means for employing effective thermal-control measures, respectively. With human assistance, the Moon offers the opportunity to establish very-large serviced structures, e.g., Arecibo-like antennas. Such structures are notoriously difficult to place in space as free-flyers.

\subsection{ELECTROMAGNETIC WAVES}

Large, Earth-based telescopes furnish the models.

\subsection{GRAVITATIONAL WAVES}

Although sensible lunar designs can be produced, it does not seem likely that in the near term the Moon will play a significant role in the channel of information.

\subsection{COSMIC RAYS}

Cherry in [5] points out some advantages of using a lunar location for particle physics and cosmicray studies: near vacuum implies lack of secondary particles and lack of attenuation; acces for low-energy particles due to the Moon's dilute magnetic enviroment; possibility of large (area, volume) detectors.

\subsection{NEUTRINOS}

There is a diverse menu of possibilities. The use of the entire Moon as a neutrino detector (Wilson in [4], which just is one approach) and the lack of a lunar atmosphere (cosmic rays interacting with the Earth's atmosphere produce a diffuse background of neutrinos) point to aspects of the Moon that lend themselves to utilizing this channel of information. Learned in [4] seconds the use of the Moon for high-energy (TeV) neutrino astronomy: "It seems possible that the future of very high cnergy neutrino astronomy is on the Moon." 


\section{A Note on the Astronomical Use of Asteroids}

Near-Earth asteroids do not present the gravity-well problem of the Moon. However, they cannot support very long baseline operations, and one cannot be assured of stable rotational behavior. Knowledge of the spin rates and orientations of axes is growing rapidly through analyses of light curves and, for closer asteroids, radar observations. Small bodies may exhibit chaotic dynamical behavior, so one would have to design an investigation that would be compatible with stochastic variations in pointing. As one goes further from the Sun, zodiacal emission decreases: over a broad range of wavelengths, the emissions is 30-100 times fainter at 3 AU than at $1 \mathrm{AU}$. An intriguing option would be a facility on a Centaur, an object with semimajor axis between Jupiter and Neptune. However, one must beware of possible eruptive behavior (but see Brown \& Luu [10] for some comfort with regard to Centaur $1995 \mathrm{GO}$ ). Caveat transgressor.

\section{Summary}

A continual flow of proposals increases the likelihood of finding some which might fill lunar niches. If dependent emplacements are to be considered, designs for connectivity and data on survivability should be pursued. The economics of astronomical facilities attached to larger lunar bases needs to be better understood. Science and mission scenarios for asteroids are needed before viability can be assessed.

\section{Acknowledgements}

The Lunar Observatory Steering Group was chartered in 1994 by Dr. Carl Pilcher, Assistant Associate Adminstrator in NASA's Office of Space Science, and he has continued his support and interest in scientific uses of the Moon. The work described in this paper was carried out by the Jet Propulsion Laboratory, California Institute of Technology, under contract with the National Acronautics and Space Adminstration.

\section{References}

1. Richardson, R. S. (May 1947), "Astronomical Observations From the Moon", Astronomical Society of the Pacific, Leaflet No.219, pp.1-8.

2. Mendell, W. W. (ed.) (1985), Lunar Bases and Space Activities of the 21st Century, Lunar and Planetary Institute, Houston, Texas.

3. Burns, J. O. and Mendell, W. (eds.) (1986), Future Astronomical Observatories on the Moon, NASA Conference Publication 2489.

4. Potter, A. E. and Wilson, T. L. (eds.) (1990), Physics and Astrophysics From a Lunar Base, AIP Conference Proceedings 202, American Institute of Physics, New York.

5. Mumma, M. J. and Smith, H. J. (1990), Astrophysics From the Moon, AIP Conference Proceeding 207, American Institute of Physics, New York.

6. Mission To The Moon (1992), ESA SP-1150, European Space Agency, Noordwijk.

7. Saunders, R. S., Pilcher, C. B., Kaplan, M. S. and McLaughlin, W. I. (1995), "The Moon as an Observational Platform for Astronomy - Long Term Strategy for a Return to the Moon", IAF-95-Q.2.05, 46th International Astronautical Conference, Oslo, Norway.

8. "Kilometric Baseline Space Interferometry: Comparison of free-flyer and moon-based versions" (June 1996), ESA SCI 96, 7.

9. McLaughlin, W. I. (1996), "The Moon is Not Space", AIAA-96-0927, 34th Aerospace Sciences Meeting \& Exhibit, January 15-18, Reno, Nevada.

10. Brown, W. R. and Luu, J. X. (1997), "CCD Photmetry of the Centaur 1995 GO", Icarus 126, pp. $218-224$. 